\title{
Efficacy and safety of an herbal formula (KBMSI-2) in the treatment of erectile dysfunction: A preliminary clinical study
}

\author{
Nam Cheol Park ${ }^{1,2}$, Sae Woong Kim ${ }^{3}$, Sung Yeoun Hwang ${ }^{4}$, Hyun Jun Park ${ }^{1,2}$ \\ ${ }^{1}$ Department of Urology, Pusan National University School of Medicine, Busan, ${ }^{2}$ Medical Research Institute of Pusan National University Hospital, Busan, ${ }^{3}$ Catholic \\ Integrative Medicine Research Institute, College of Medicine, The Catholic University of Korea, Seoul, ${ }^{4}$ Korea Bio Medical Science Institute, Seoul, Korea
}

Purpose: To investigate the efficacy and safety of KBMSI-2, an herbal formula consisting of Ginseng Radix Rubra, Dioscorea tenuipes, Cornus officinalis Sieb. et Zucc., Lycium chinense Mill, and Curcuma longa Linn, for the treatment of erectile dysfunction (ED). Materials and Methods: Patients were instructed to take placebo or $6 \mathrm{~g}$ of KBMSI-2 twice per day for 8 weeks, at least 1 hour after food intake. The primary outcome was a change from baseline in erectile function (EF) domain scores of the International Index of Erectile Function (IIEF) questionnaire. Secondary outcome included changes from baseline in all domain scores of the IIEF, scores on the Aging Males' Symptoms scale, and serum total testosterone levels, as well as changes in questions 2 and 3 of the Sexual Encounter Profile, responses to the Global Assessment Question, and changes in the number of 'yes' responses on the Androgen Deficiency in Aging Males questionnaire.

Results: Patients receiving KBMSI-2 had a statistically significant improvement in baseline IIEF-EF domain scores at 8 weeks compared to the placebo group. Intercourse satisfaction domain and the total IIEF scores also increased in the KBMSI- 2 group. However, we could not find any significant differences in other efficacy variables between the groups. Only one patient had an adverse event, which was mild in severity.

Conclusions: This preliminary clinical study of KBMSI-2 shows significant improvements in EF and intercourse satisfaction, as measured by the IIEF in patients with ED. Further studies using a larger number of patients in the long term should follow.

Keywords: Dioscorea; Erectile dysfunction; Ginseng; Herbal medicine; Phytotherapy

This is an Open Access article distributed under the terms of the Creative Commons Attribution Non-Commercial License (http://creativecommons.org/licenses/by-nc/4.0) which permits unrestricted non-commercial use, distribution, and reproduction in any medium, provided the original work is properly cited.

\section{INTRODUCTION}

Erectile dysfunction (ED) is defined as the persistent inability to attain or maintain penile erection sufficient for sexual intercourse [1]. Phosphodiesterase type 5 (PDE5) inhibitors have been the most commonly prescribed medications for ED, and include sildenafil citrate, tadalafil, vardenafil, udenafil, mirodenafil, and avanafil. Although PDE5 inhibitors are efficacious in the treatment of ED, high rates of treatment discontinuation have been reported,

Received: 29 October, 2018 - Accepted: 25 March, 2019

Corresponding Author: Hyun Jun Park

Department of Urology, Pusan National University School of Medicine, Medical Research Institute of Pusan National University Hospital, 179 Gudeok-ro, Seogu, Busan 49241, Korea

TEL: +82-51-240-7347, FAX: +82-51-247-5443, E-mail: joon501@naver.com

ORCID: https://orcid.org/0000-0003-0566-9574 
ranging from $14 \%$ to $80.4 \%[2,3]$. Reasons for treatment discontinuation include lack of efficacy, adverse events (AEs), fear of AEs, and the inconvenience of waiting for the onset of drug of action [4,5]. If first-line ED therapy, such as the use of PDE5 inhibitors, is not sufficient, a secondline of therapy is needed. The most commonly used secondline therapy is intracavernous injection [6], which has a success rate of about $70 \%$ to $85 \%$. However, poor treatment compliance and ensuing high dropout rates (41\%-68\%) have been reported $[7,8]$.

Additionally, the daily treatment with PDE5 inhibitors, which is expected to reduce the pathologic progression of $\mathrm{ED}$, failed to show consistent positive effects in many studies [9]. In addition, unassisted erectile function (EF) was not improved after cessation of active therapy [10]. Therefore, there is an increasing need to develop drugs that function differently from PDE5 inhibitors and can improve EF when taken continuously.

The major components of KBMSI-2 are Ginseng Radix Rubra, Dioscorea tenuipes, Cornus officinalis Sieb. et Zucc., Lycium chinense Mill, and Curcuma longa Linn. These ingredients are widely known plants and compounds used by the general population. Their extracts, when used individually or in combination, are effective in triggering penile erection in white rats that have the same erectile mechanism as humans [11]. Yoon et al. [12] reported that the peak intracavernosal pressure/mean arterial pressure (ICP/MAP) ratio, endothelial nitric oxide synthase (eNOS), neuronal NOS (nNOS), and cyclic guanosine monophosphate (cGMP) expression in rats treated with KBMSI-2 increased, and the smooth muscle component increased when analyzed by Masson's trichrome staining. They suggested that KBMSI-2 improved the EF by preserving the smooth muscle content and inhibiting fibrosis of the corpus cavernosum in a streptozotocin (STZ)-induced diabetic rat model. Based on these findings, we conducted this preliminary clinical study to determine whether the therapeutic effect of KBMSI-2 on ED observed in animal studies was valid in humans.

\section{MATERIALS AND METHODS}

\section{Ethics statement and description of partici- pants}

This was a double-blind, randomized, placebo-controlled, parallel-group study. The present study protocol was reviewed and approved by the Institutional Review Board of Pusan National University Hospital (approval number: H-1201-005-001). Written informed consent was obtained from each patient before randomization. The protocol was registered in ClinicalTrials.gov (NCT identifier: NCT02413099). We included only male patients because the primary purpose of this study was evaluating the EFs.

\section{Study design}

Initially, eligible patients had a 4-week, treatmentfree run-in period during which they were required to have attempted intercourse on at least 4 separate days, experiencing failure in at least half of these attempts. Subsequently, patients were randomly assigned to receive the investigational products (6 $\mathrm{g}$ of KBMSI-2 or placebo) twice per day for 8 weeks, at least 1 hour after food intake (Fig. 1). During the 8-week treatment period, patient tolerance, and responses to the investigational drugs were assessed every 4 weeks, and follow-up contact was made 6 or 7 days after the 8-week treatment to evaluate any additional AEs.

\section{Subjects}

Inclusion criteria were as follows: 1) men with a history of ED for at least 6 months, according to the National Institutes of Health (NIH) Consensus Statement (inability to attain and/or maintain penile erection sufficient for satisfactory sexual performance); 2) EF domain scores on the International Index of Erectile Function (IEF) questionnaire $\leq 25$; 3) age range between 40 and 80 years old; and 4) involvement in a stable, monogamous relationship with a female sexual partner, with a failure rate $>50 \%$ of at least four intercourse attempts during the 4-week run-in period.

Men with the following conditions were excluded from the study: penile anatomical deformity, spinal cord injury, radical prostatectomy, pelvic organ surgery, diagnosis of another sexual disorder, uncontrolled diabetes mellitus $\left(\mathrm{HbA}_{1 \mathrm{C}}>12 \%\right)$, serum creatinine $>2.5 \mathrm{mg} / \mathrm{dL}$, an uncontrolled psychiatric disorder, history of major hematological, renal, or hepatic abnormalities, recent (within the previous 6 months)

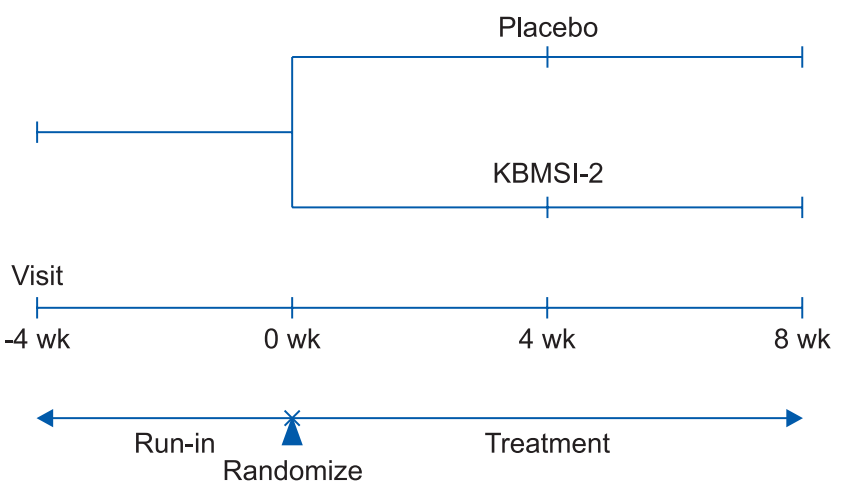

Fig. 1. Trial schedule. 
history of cardiovascular disease, stroke or myocardial infarction, cardiac failure, unstable angina, life-threatening arrhythmia, and a history of alcoholism or substance abuse. Patients who had taken PDE5 inhibitors within 2 weeks or testosterone replacement therapy in the previous 12 months were also excluded. Concomitant use of any ED treatment was prohibited.

\section{Investigational drugs}

KBMSI-2 capsules (6 g/capsule) and placebo was provided by the Korea Bio Medical Science Institute (Seoul, Korea). KBMSI-2 was prepared with Ginseng Radix Rubra, $C$. officinalis Sieb. et Zucc., L. chinense Mill, D. tenuipes, and $C$. longa Linn, and was analyzed by high-performance liquid chromatography. KBMSI-2 contained the following ginsenosides: $260.53 \mu \mathrm{g} / \mathrm{g} \mathrm{Rb} 1,543.91 \mu \mathrm{g} / \mathrm{g}$ Rb2, $424.92 \mu \mathrm{g} / \mathrm{g}$ Rc, $377.32 \mu \mathrm{g} / \mathrm{g}$ Re, 1,160.55 $\mu \mathrm{g} / \mathrm{g}$ Rf, and $703.97 \mu \mathrm{g} / \mathrm{g}$ Rg1, in addition to $60.73 \mu \mathrm{g} / \mathrm{g}$ curcumin, $98.66 \mu \mathrm{g} / \mathrm{g}$ allantoin, and $744.13 \mu \mathrm{g} / \mathrm{g}$ loganin. The placebo capsules were identical in shape, color, and taste.

\section{Outcome measures}

The primary efficacy outcome was the change in EF domain scores from baseline on the IIEF questionnaire, which was calculated by comparing total scores from questions 1 to 5 and 15 [13]. Secondary efficacy outcomes included changes from baseline IIEF scores in all other domains of the questionnaire. Changes from baseline in question 2 (Were you able to insert your penis into your partner's vagina?) and 3 (Did your erection last long enough for you to have successful intercourse?) of the Sexual Encounter Profile (SEP), patient responses to the Global Assessment Question (GAQ: Has the treatment you have been taking during the last 4 weeks improved your erection?), changes in the number of 'yes' responses on the Androgen Deficiency in Aging Males (ADAM) questionnaire [14], and changes from baseline on the Aging Males' Symptoms (AMS) scale [15] was also analyzed. In addition, serum total testosterone levels were checked at baseline and at the end of the study.

Penile color Doppler ultrasonography was performed by conventional techniques in supine position, after achieving erection by administering intracavernosal injection with 5- to $10-\mathrm{MHz}$ linear probe at baseline and at the end of the study. Peak systolic velocity (PSV) and end diastolic velocity (EDV) measurements were performed in both cavernous arteries at the penoscrotal junction. Measurements were repeated at 5-minute intervals for 20 to 30 minutes and the most accurately measured right/left mean PSV and EDV values were recorded.

AEs were classified as adverse changes from baseline that occurred throughout the study period. Safety assessments included laboratory tests (hematology, clinical biochemistry, blood coagulation test, and urinalysis), vital signs (blood pressure and heart rate), physical examination, 12-lead electrocardiogram recordings, and patient-reported AEs.

\section{Statistical methods}

Efficacy of KBMSI-2 was based on the analysis of all the data obtained. Repeated measures Analysis of Covariance (ANCOVA), with baseline characteristics as covariates, was used to evaluate the significance of primary and secondary measures of efficacy, except for GAQ. Efficacy was calculated relative to the placebo group and differences from baseline at given points, including the end of treatment, were analyzed. Repeated measures Analysis of Variance (ANOVA), was used to compare the differences between placebo and KBMSI-2 treated patients, at specific time points relative to baseline. Inter-group differences in GAQ response rate were analyzed using the $\chi^{2}$ test. Statistical significance was considered at a $p<0.05$. The number of patients evaluated in this study was based on data obtained from the IEF$\mathrm{EF}$ domain from a previous study with an herbal formula [16]. According to the standards of a per-protocol (PP) analysis, 18 valid patients were required for each group. Assuming a 20\% dropout rate, a total of 44 randomized patients (22 per group) were required for effective analysis. Changes from baseline for the continuous safety variables, including the results of laboratory analysis and vital signs, were evaluated by ANOVA. Inter-group comparisons of treatment-emergent AE frequencies were conducted using a $\chi^{2}$ test or Fisher's exact test. Baseline demographics in the KBMSI-2 and placebo groups were analyzed by ANOVA for continuous variables, and $\chi^{2}$ test was used to analyze categorical variables. Block randomization generated by SAS Proc Plan procedure was utilized. Statistical analyses were performed using SAS ver. 9.1 (SAS Institute, Cary, NC, USA).

\section{RESULTS}

\section{Demographics}

In total, 44 Korean men completed baseline evaluations and were randomly assigned to receive placebo or $6 \mathrm{~g}$ of KBMSI-2. Among the intention to treat population, 41 patients completed the study, and 26 constituted the PP population (Fig. 2). At baseline, no clinically or statistically significant differences in demographics or clinical variables 
were found between the treatment groups (Table 1).

\section{Primary efficacy outcome variable}

\section{1) IIEF-EF domain}

In the analysis of differences, the KBMSI-2 group showed a statistically significant improvement relative to baseline in the IIEF-EF domain scores at 4 and 8 weeks. However, no significant improvement was observed in the placebo group (Fig. 3).

\section{Secondary efficacy outcome variable}

\section{1) IIEF-all domains (Table 2)}

Compared to baseline, there were significant improvements in all domains of the IIEF questionnaire after 4 weeks of KBMSI-2 treatment. However, there was no change in the placebo group. When comparing the changes between the two groups, the results of all analyses except that of overall satisfaction showed significant differences. At the end of the study, there were significant improvements in the intercourse satisfaction domain of the IIEF and the total IIEF score in the KBMSI-2 group relative to baseline.

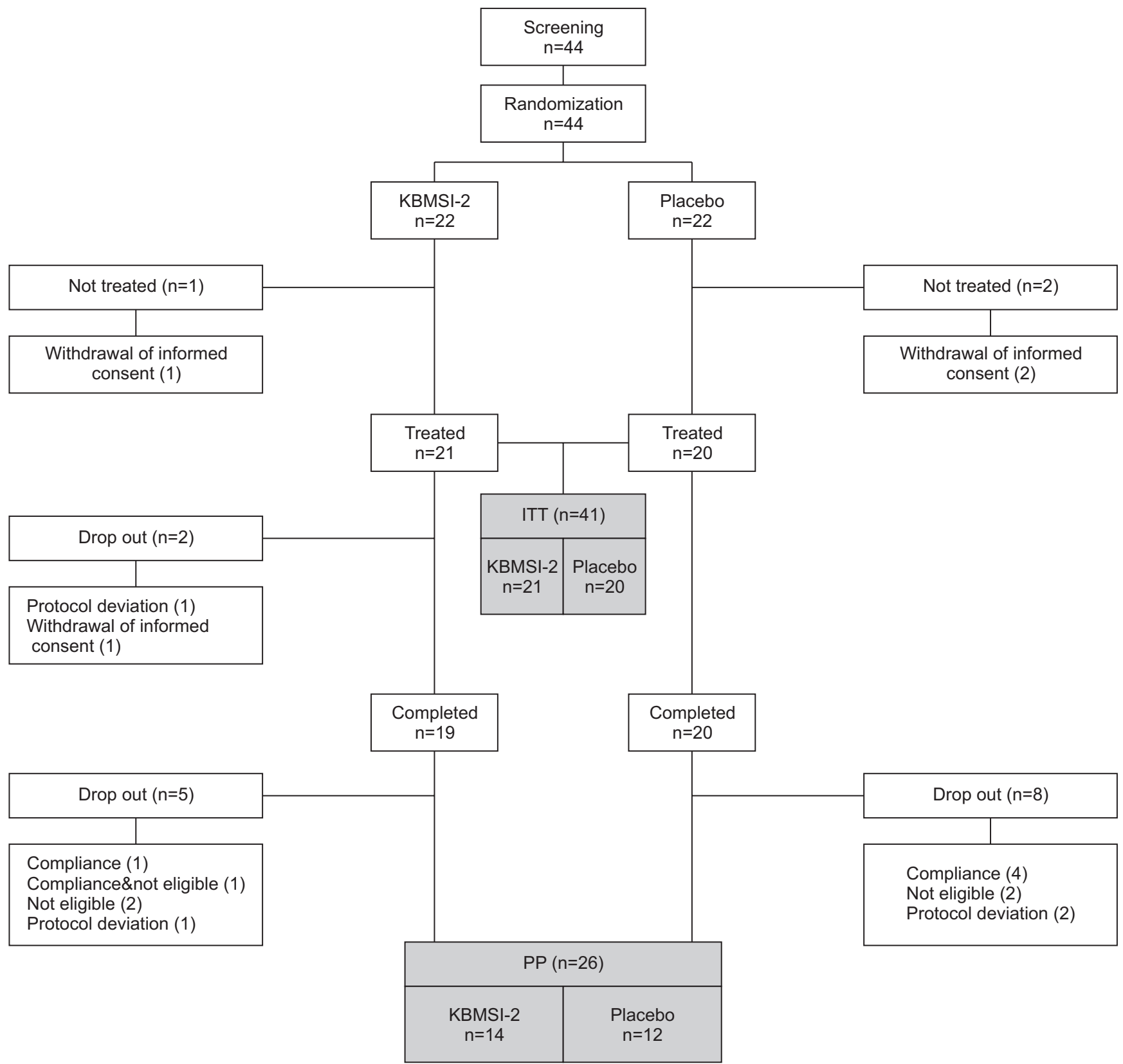

Fig. 2. Trial flow chart. ITT, intention to treat; PP, per-protocol. 
Table 1. Baseline characteristics of patients

\begin{tabular}{|c|c|c|c|c|c|c|}
\hline \multirow{2}{*}{ Characteristic } & \multicolumn{2}{|c|}{ ITT $(n=41)$} & \multirow{2}{*}{$p$-value ${ }^{a}$} & \multicolumn{2}{|c|}{$P P(n=26)$} & \multirow{2}{*}{ p-value ${ }^{a}$} \\
\hline & KBMSI-2 $(n=21)$ & Placebo $(n=20)$ & & KBMSI-2 $(n=14)$ & Placebo $(n=12)$ & \\
\hline Smoking & & & $0.0478^{b}$ & & & $0.2031^{b}$ \\
\hline Non-smoker & $21(100.0)$ & $16(80.0)$ & & $14(100.0)$ & $10(83.3)$ & \\
\hline Smoker & $0(0.0)$ & $4(20.0)$ & & $0(0.0)$ & $2(16.7)$ & \\
\hline Alcohol consumption & & & $0.4123^{b}$ & & & $0.6709^{b}$ \\
\hline Yes & $11(52.4)$ & $13(65.0)$ & & $7(50.0)$ & $7(58.3)$ & \\
\hline No & $10(47.6)$ & $7(35.0)$ & & $7(50.0)$ & $5(41.7)$ & \\
\hline Age (y) & $55.43 \pm 9.61(35-73)$ & $57.70 \pm 7.87(38-71)$ & 0.4142 & $59.14 \pm 7.54(47-74)$ & $58.75 \pm 7.36(46-72)$ & 0.8946 \\
\hline Duration of ED (y) & $3.89 \pm 2.58(1-10)$ & $5.62 \pm 3.74(0.83-13)$ & 0.0913 & $4.46 \pm 2.53(2-10)$ & $5.11 \pm 2.88(1-10)$ & 0.5480 \\
\hline Category of ED & & & $0.5231^{\mathrm{b}}$ & & & $0.7690^{b}$ \\
\hline Arteriogenic & $17(81.0)$ & $13(65.0)$ & & $12(85.7)$ & $9(75.0)$ & \\
\hline Venogenic & $3(14.3)$ & $6(30.0)$ & & $2(14.3)$ & $3(25.0)$ & \\
\hline Psychogenic & $1(4.8)$ & $1(5.0)$ & & $0(0.0)$ & $0(0.0)$ & \\
\hline Medical history & & & $0.4311^{\mathrm{b}}$ & & & $0.6707^{b}$ \\
\hline Diabetes mellitus & $9(42.9)$ & $8(40.0)$ & & $7(50.0)$ & $7(58.3)$ & \\
\hline Hypertension & $7(33.3)$ & $8(40.0)$ & & $6(42.9)$ & $5(41.7)$ & \\
\hline Dyslipidemia & $5(23.8)$ & $3(15.0)$ & & $4(28.6)$ & $3(25.0)$ & \\
\hline \multicolumn{7}{|l|}{ IIEF } \\
\hline Erectile function & $14.43 \pm 7.18$ & $15.05 \pm 6.72$ & 0.7766 & $14.86 \pm 6.36$ & $16.58 \pm 5.84$ & 0.4807 \\
\hline Intercourse satisfaction & $5.38 \pm 2.69$ & $6.15 \pm 3.41$ & 0.4263 & $5.71 \pm 2.55$ & $6.92 \pm 2.91$ & 0.2725 \\
\hline Orgasmic function & $4.67 \pm 2.61$ & $5.30 \pm 3.26$ & 0.4957 & $5.00 \pm 2.18$ & $6.17 \pm 3.10$ & 0.2730 \\
\hline Sexual desire & $5.10 \pm 1.51$ & $5.80 \pm 1.77$ & 0.1771 & $5.21 \pm 1.37$ & $6.00 \pm 1.81$ & 0.2200 \\
\hline Overall satisfaction & $5.29 \pm 1.79$ & $6.15 \pm 1.31$ & 0.0870 & $5.57 \pm 1.74$ & $6.08 \pm 1.31$ & 0.4122 \\
\hline Total & $34.86 \pm 14.65$ & $38.45 \pm 14.87$ & 0.4405 & $36.36 \pm 12.82$ & $41.75 \pm 12.91$ & 0.2971 \\
\hline \multicolumn{7}{|l|}{ Sexual Encounter Profile } \\
\hline Question 2 & $75.00 \pm 42.15$ & $76.79 \pm 40.99$ & 0.8927 & $77.50 \pm 32.15$ & $83.33 \pm 38.92$ & 0.7675 \\
\hline Question 3 & $52.50 \pm 44.35$ & $49.95 \pm 45.86$ & 0.8592 & $51.43 \pm 37.80$ & $52.50 \pm 48.45$ & 0.2743 \\
\hline Aging Males' Symptoms score & $38.00 \pm 12.07$ & $36.55 \pm 14.51$ & 0.7293 & $41.14 \pm 12.15$ & $40.00 \pm 16.81$ & 0.8426 \\
\hline ADAM (no. of positive response) & $5.24 \pm 2.19$ & $5.05 \pm 2.39$ & 0.7941 & $5.86 \pm 1.66$ & $5.17 \pm 2.17$ & 0.3668 \\
\hline Total testosterone (ng/mL) & $4.43 \pm 1.57$ & $4.29 \pm 1.35$ & 0.7577 & $4.69 \pm 1.65$ & $4.41 \pm 1.50$ & 0.6587 \\
\hline Peak systolic velocity $(\mathrm{cm} / \mathrm{s})$ & $10.05 \pm 5.05$ & $11.61 \pm 4.55$ & 0.5937 & $10.23 \pm 5.60$ & $12.14 \pm 4.17$ & 0.3398 \\
\hline End diastolic velocity $(\mathrm{cm} / \mathrm{s})$ & $3.68 \pm 1.80$ & $5.85 \pm 3.14$ & 0.0114 & $3.91 \pm 2.00$ & $5.82 \pm 3.03$ & 0.0665 \\
\hline
\end{tabular}

Values are presented as number (\%), mean \pm SD (minimun-maximum), or mean \pm SD only.

ITT, intention to treat; PP, per-protocol; ED, erectile dysfunction; IIEF, International Index of Erectile Function; ADAM, Androgen Deficiency in Aging Males; SD, standard deviation.

${ }^{\mathrm{a}}$ :t-test or chi-square test, ${ }^{\mathrm{b}}$ :Fisher's exact test.

However, no changes in the placebo group were observed. Additionally, changes between the groups were statistically significant.

\section{2) SEP 2 and 3 (Fig. 4)}

The proportions of "yes" responders were not changed in SEP 2 (77.50 \pm 32.15 to $80.95 \pm 38.60)$ and SEP $3(51.43 \pm 37.80$ to $71.00 \pm 40.98$ after 8 weeks of treatment) in the KBMSI-2 group. There were no differences compared to the placebo group.

\section{3) GAQ (Fig. 5)}

The proportion of patient who responded "yes" to the GAQ at the end of study was $50.0 \%$ and $58.3 \%$ in the KBMSI-2 and placebo groups, respectively. This difference was not statistically significant.

\section{4) AMS total score (Table 3 )}

AMS total score decreased in the KBMSI-2 group at the end of study (41.14 \pm 12.15 to $35.43 \pm 14.34$ ). However, the change was not statistically significant compared to the placebo group. 


\section{5) ADAM questionnaire}

The number of 'yes' responses to the ADAM questionnaire decreased in the KBMSI-2 group at the end of study (5.86 \pm 1.66 to $4.86 \pm 2.18$ ). However, the change was not significant compared to the placebo group.

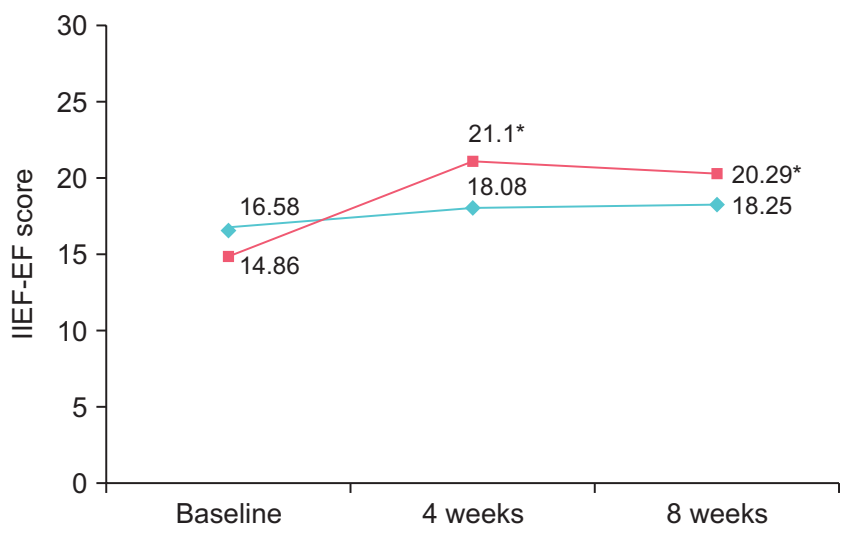

Fig. 3. Effects of KBMSI-2 on International Index of Erectile FunctionErectile Function (IIEF-EF) score. ${ }^{*} p<0.01$ vs. baseline.

\section{6) PSV and EDV (Table 4)}

PSV significantly increased in the KBMSI-2 group at the end of the study relative to baseline $(10.23 \pm 5.60 \mathrm{~cm} / \mathrm{s}$ to $13.64 \pm 5.99 \mathrm{~cm} / \mathrm{s} ; \mathrm{p}=0.0240$ ). However, this change was not significant compared to the change observed in the placebo group ( $3.41 \pm 5.00 \mathrm{~cm} / \mathrm{s}$ vs. $2.97 \pm 3.51 \mathrm{~cm} / \mathrm{s} ; \mathrm{p}=0.7974)$. There was no change in EDV and resistive index (RI) in either group.

\section{7) Serum total testosterone levels}

There were no changes in total levels of serum testosterone in either group $(4.69 \pm 1.65 \mathrm{ng} / \mathrm{mL}$ to $4.09 \pm 1.16 \mathrm{ng} / \mathrm{mL}$; $\mathrm{p}=0.1624$ ).

\section{8) Safety and tolerability}

To analyze the safety of KBMSI-2, 41 subjects who took at least one dose were included. Only one patient in the KBMSI-2 group reported a mild itching sensation during the study and in the follow-up period. No clinically significant change in laboratory tests, electrocardiogram, or blood pressure was observed in either group.

Table 2. International Index of Erectile Function (IIEF) domain score

\begin{tabular}{|c|c|c|c|c|c|c|c|}
\hline $\begin{array}{l}\text { Treatment } \\
\text { group }\end{array}$ & Baseline & 4 weeks & $\begin{array}{c}\text { Difference } \\
\text { (4 wk-baseline) }\end{array}$ & $p$-value ${ }^{a}$ & 8 weeks & $\begin{array}{c}\text { Difference } \\
\text { (8 wk-baseline) }\end{array}$ & $p$-value \\
\hline \multicolumn{8}{|l|}{ Erectile function } \\
\hline KBMSI-2 $(n=14)$ & $14.86 \pm 6.36$ & $21.14 \pm 5.55$ & $6.29 \pm 6.34$ & 0.0026 & $20.29 \pm 8.10$ & $5.43 \pm 6.00$ & 0.0049 \\
\hline \multirow[t]{2}{*}{ Placebo $(n=12)$} & $16.58 \pm 5.84$ & $18.08 \pm 8.06$ & $1.50 \pm 5.11$ & 0.3309 & $18.25 \pm 7.74$ & $1.67 \pm 3.03$ & 0.0828 \\
\hline & & $p$-value ${ }^{c}$ & 0.0470 & & $p$-value ${ }^{d}$ & 0.0527 & \\
\hline \multicolumn{8}{|c|}{ Intercourse satisfaction } \\
\hline KBMSI-2 & $5.71 \pm 2.55$ & $8.21 \pm 2.29$ & $2.50 \pm 1.99$ & 0.0004 & $7.64 \pm 3.08$ & $1.93 \pm 2.02$ & 0.0034 \\
\hline \multirow[t]{2}{*}{ Placebo } & $6.92 \pm 2.91$ & $7.58 \pm 3.65$ & $0.67 \pm 2.02$ & 0.2761 & $6.83 \pm 4.00$ & $-0.08 \pm 2.57$ & 0.9127 \\
\hline & & $p$-value $e^{c}$ & 0.0287 & & $p$-value ${ }^{d}$ & 0.0351 & \\
\hline \multicolumn{8}{|l|}{ Orgasmic function } \\
\hline KBMSI-2 & $5.00 \pm 2.18$ & $7.14 \pm 2.07$ & $2.14 \pm 1.92$ & 0.0011 & $6.57 \pm 3.01$ & $1.57 \pm 3.08$ & 0.0787 \\
\hline \multirow[t]{2}{*}{ Placebo } & $6.17 \pm 3.10$ & $6.25 \pm 3.11$ & $0.08 \pm 2.11$ & 0.8936 & $6.00 \pm 3.52$ & $-0.17 \pm 2.33$ & 0.8088 \\
\hline & & $p$-value ${ }^{c}$ & 0.0154 & & $p$-value ${ }^{d}$ & 0.1228 & \\
\hline \multicolumn{8}{|l|}{ Sexual desire } \\
\hline KBMSI-2 & $5.21 \pm 1.37$ & $6.64 \pm 1.15$ & $1.43 \pm 1.34$ & 0.0016 & $6.07 \pm 1.94$ & $0.86 \pm 2.14$ & 0.1584 \\
\hline \multirow[t]{2}{*}{ Placebo } & $6.00 \pm 1.81$ & $5.92 \pm 2.07$ & $-0.08 \pm 1.00$ & 0.7774 & $6.00 \pm 1.60$ & $0.00 \pm 1.71$ & 1.0000 \\
\hline & & $p$-value ${ }^{c}$ & 0.0037 & & $p$-value ${ }^{d}$ & 0.2761 & \\
\hline \multicolumn{8}{|l|}{ Overall Satisfaction } \\
\hline KBMSI-2 & $5.57 \pm 1.74$ & $6.64 \pm 1.74$ & $1.07 \pm 1.64$ & 0.0294 & $6.57 \pm 2.17$ & $1.00 \pm 2.18$ & 0.1104 \\
\hline \multirow[t]{2}{*}{ Placebo } & $6.08 \pm 1.31$ & $5.92 \pm 2.27$ & $-0.17 \pm 2.33$ & 0.8088 & $6.25 \pm 2.01$ & $0.17 \pm 1.80$ & 0.7545 \\
\hline & & $p$-value ${ }^{c}$ & 0.1260 & & $p$-value ${ }^{d}$ & 0.3041 & \\
\hline \multicolumn{8}{|l|}{ IIEF total } \\
\hline KBMSI-2 & $36.36 \pm 12.82$ & $49.79 \pm 11.00$ & $13.43 \pm 11.26$ & 0.0006 & $47.14 \pm 17.24$ & $10.79 \pm 13.80$ & 0.0118 \\
\hline \multirow[t]{2}{*}{ Placebo } & $41.75 \pm 12.91$ & $43.75 \pm 18.35$ & $2.00 \pm 10.92$ & 0.5388 & $43.33 \pm 16.59$ & $1.58 \pm 8.08$ & 0.5115 \\
\hline & & $p$-value ${ }^{c}$ & 0.0151 & & $p$-value ${ }^{d}$ & 0.0539 & \\
\hline
\end{tabular}

Values are presented as mean \pm standard deviation.

${ }^{a}$ :Paired t-test for comparison of difference (4 weeks) from baseline. ${ }^{b}$ :Paired t-test for comparison of difference ( 8 weeks) from baseline. ${ }^{c}: t-t e s t$ for comparison of difference ( 4 weeks) between groups. ${ }^{\mathrm{d}}$ :t-test for comparison of difference ( 8 weeks) between groups. 

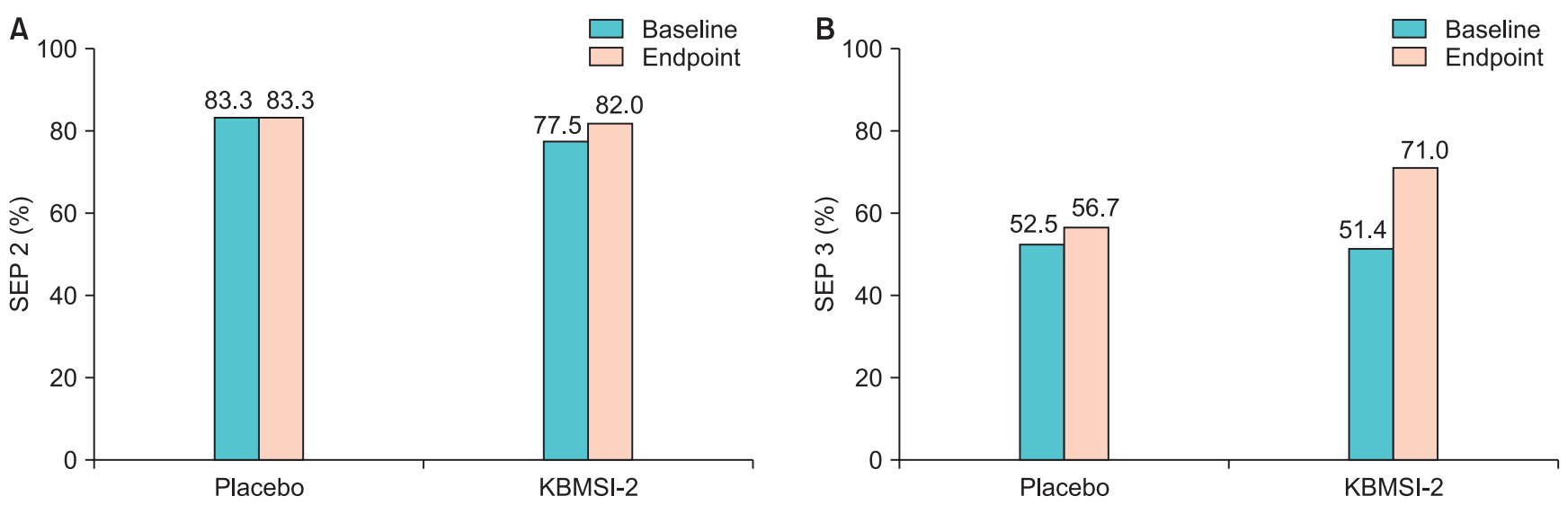

Fig. 4. Effects of KBMSI-2 on the responses to SEP 2 (A) and SEP 3 (B). SEP 2, Sexual Encounter Profile Question 2; SEP 3, Sexual Encounter Profile Question 3.

Table 3. Aging Males' Symptoms (AMS) scale

\begin{tabular}{ccccc}
\hline Treatment group & Baseline & $\mathbf{8}$ weeks & Difference & p-value $^{\text {a }^{2}}$ \\
\hline AMS score & & & & 0.1019 \\
KBMSI-2 & $41.14 \pm 12.15$ & $35.43 \pm 14.34$ & $-5.71 \pm 12.15$ & 0.5998 \\
Placebo & $40.00 \pm 16.81$ & $42.42 \pm 11.68$ & $2.42 \pm 15.49$ & 0.1468 \\
\end{tabular}

Values are presented as mean \pm standard deviation.

${ }^{a}$ :Paired t-test for comparison of difference from baseline. ${ }^{b}$ :t-test for comparison of difference between groups.

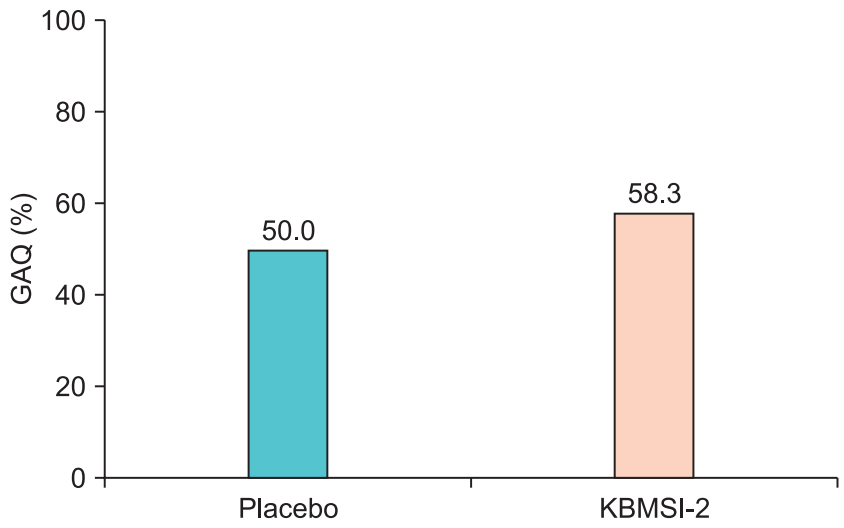

Fig. 5. Global Assessment Question (GAQ) after treatment.

\section{DISCUSSION}

Herbs known to be effective in improving sexual function or in the treatment of ED have been used in traditional medicine as extracts, herbal teas, or topical agents, and in modern western medicine to produce drugs by separating or synthesizing the active ingredients chemically [17]. Many natural substances used by the indigenous populations of Asia, Africa, and the Americas have provided ideas for drug development, and laid the groundwork for the integration of western and oriental medicine [17,18]. The herbal formula (KBMSI-2) used in this study was prepared with Ginseng Radix Rubra, C. officinalis Sieb. et Zucc., L. chinense Mill,
$D$. tenuipes, and $C$. longa Linn. The active ingredients in KBMSI-2 are believed to be ginsenosides, curcumin, allantoin, and loganin. Korean red ginseng (unskinned Panax ginseng, before it is steamed or otherwise heated and subsequently dried) is one of the most widely used herbal remedies, and its potential mechanism of action is via hormonal regulation, similar to that of testosterone. However, our data showed that total testosterone levels did not change, although several other studies appear to refute this hypothesis [19,20]. Another suggestion is that red ginseng might induce relaxation of the smooth muscles of the corpus cavernosum, via the nitric oxide (NO) pathway [21,22]. Ginsenosides, which are thought to be the major active ingredients in red ginseng, result in a dose-dependent relaxation of the corpus cavernosal smooth muscle in rabbits by increasing the release of NO [22-24]. Curcumin derivatives mediate erection via upregulation of the heme oxygenase-1 gene and cyclic guanosine monophosphate. Curcumin may also be involved in the upregulation of gene expression of eNOS, nNOS, and nuclear transcription factorerythroid 2, and the downregulation of NF- $\kappa \beta$, p38, and inducible NOS gene expression [25,26]. In a clinical study using a novel curcumin derivative, in combination with tadalafil or sildenafil, EF was significantly enhanced and a prolonged duration of action was observed [27,28]. Allantoin is contained in $D$. tenuipes as a major active compound. In 
Table 4. Peak systolic velocity (PSV) and end diastolic velocity (EDV)

\begin{tabular}{|c|c|c|c|c|}
\hline Treatment group & Baseline & 8 weeks & Difference & p-value ${ }^{a}$ \\
\hline \multicolumn{5}{|l|}{ PSV $(\mathrm{cm} / \mathrm{s})$} \\
\hline KBMSI-2 & $10.23 \pm 5.60$ & $13.64 \pm 5.99$ & $3.41 \pm 5.00$ & 0.0240 \\
\hline \multirow[t]{2}{*}{ Placebo } & $12.14 \pm 4.17$ & $15.11 \pm 5.28$ & $2.97 \pm 3.51$ & 0.0138 \\
\hline & & $p$-value ${ }^{b}$ & 0.7974 & \\
\hline \multicolumn{5}{|l|}{$\operatorname{EDV}(\mathrm{cm} / \mathrm{s})$} \\
\hline KBMSI-2 & $3.91 \pm 2.00$ & $5.42 \pm 2.62$ & $1.51 \pm 2.53$ & 0.0535 \\
\hline \multirow[t]{2}{*}{ Placebo } & $5.82 \pm 3.03$ & $7.03 \pm 3.35$ & $1.21 \pm 2.16$ & 0.0790 \\
\hline & & $p$-value ${ }^{b}$ & 0.7458 & \\
\hline \multicolumn{5}{|l|}{ Resistive index } \\
\hline KBMSI-2 & $0.61 \pm 0.04$ & $0.60 \pm 0.09$ & $-0.01 \pm 0.01$ & 0.815 \\
\hline \multirow[t]{2}{*}{ Placebo } & $0.52 \pm 0.05$ & $0.53 \pm 0.05$ & $0.01 \pm 0.01$ & 0.779 \\
\hline & & $p$-value ${ }^{b}$ & 0.756 & \\
\hline
\end{tabular}

Values are presented as mean \pm standard deviation.

${ }^{a}$ :Paired t-test for comparison of difference from baseline. ${ }^{b}$ :t-test for comparison of difference between groups.

traditional Chinese medicine, Dioscorea is used to improve insulin resistance and prevent inflammation and ulcers [29]. Loganin is the primary bioactive component contained in $C$. officinalis Sieb. et Zucc. The herb $C$. officinalis has been used traditionally in Chinese preparations for the management of diabetes and is capable of reducing levels of some vasoactive factors and cytokines, such as NO, endothelin, and tumor necrosis factor- $\alpha$ in the diabetic state [30]. In 2013, Yoon et al. [12] reported a study investigating the effects of KBMSI-2 on $\mathrm{ED}$ in the STZ-induced diabetic rat model. In that study, the ICP/MAP ratio was increased in the KBMSI-2 treatment group compared to the untreated group. Masson's trichrome staining confirmed that the smooth muscle content was increased in the KBMSI-2 group. nNOS, eNOS, and cGMP expression levels were increased in the KBMSI-2 treatment group. These results suggest that KBMSI-2 improved EF by preserving the vascular endothelium and smooth muscle content and inhibiting fibrosis in the corpus cavernosum. The purpose of this clinical trial was to investigate the efficacy and safety of KBMSI-2 in patients with ED. KBMSI-2 showed statistically significant improvements in the IEF-EF domain scores at 4 and 8 weeks. With regard to other domains of IIEF, KBMSI-2 showed improvements in all domains of IIEF at 4 weeks and the intercourse satisfaction domain and total score at 8 weeks. These results are in accordance with the results of a previous animal study that involved KBMSI-2, which showed improvement in $\mathrm{EF}$ by preserving smooth muscle content and inhibiting fibrosis of the corpus cavernosum in a diabetic rat model [12]. However, we could not confirm any improvement in hemodynamic parameters, such as PSV, EDV, and RI. Although PSV increased in the KBMSI-2 group, the change was not significantly different from that of the placebo group. These inconsistent results are presumably due to the fact that we included the venogenic and psychogenic ED patients in this study, and we could not perform analysis according to ED etiology due to the small number of patients. In addition, 8 weeks of this study duration is too short to induce the vascular changes in the patients. A longer study duration may be needed to evaluate KBMSI2-induced changes in hemodynamic parameters, cavernosal tissues and endothelial function. Another contributing factor may be related to the contribution of the central nervous system to the improvement of $\mathrm{EF}$, which was not evaluated in this study. Interestingly, despite the improved IEF-EF, there were no significant changes in SEP 2 and SEP 3. It is necessary to evaluate why patients responded differently to similar kinds of questionnaire items.

We expected that there would be a change in total serum testosterone levels after treatment in the experimental group. However, we could not find any significant changes in the total levels of serum testosterone. This result is in contrast with the theory that ginseng, one of the main active ingredients of KBMSI-2, has hormonal effects similar to that of testosterone $[19,20]$. This may be a caveat of the normal testosterone levels observed at baseline and the short period of the study.

The present study had several limitations that should be noted. Although we identified significant improvements in the IIEF-EF domain as the primary outcome variable, and intercourse satisfaction domain and total scores in the IEF as secondary outcome variables, we did not find significant improvements in other parameters such as GAQ, AMS, and ADAM. These analyses addressed the general well- 
being and overall satisfaction of the patients, which were expected to improve. Second, because of the sociocultural differences among various populations of different ethnic origins, the efficacy and safety profile of KBMSI-2 observed in this study, which only included Korean patients, may have resulted in somewhat different outcomes from that reported in other ethnic groups. A third limitation is that this study did not use a cross-over design. Larger clinical trials that investigate the efficacy and safety of KBMSI-2 in other ethnicities and in treating ED induced by different reasons, using different dosing regimens as well as direct comparative studies with PDE5 inhibitors, are needed. Forth, the number of subjects in this study was so small that we could not analyze the results according to the degree of ED and etiology. This is why the mechanism of study drug is not sufficiently proven. Lastly, follow-up tests were not performed after a certain period of time following the completion of treatment. Therefore, we could not ascertain whether the effect of KBMSI-2 was sustained after the treatment.

\section{CONCLUSIONS}

This preliminary clinical study of an herbal formula, KBMSI-2, prepared based on Ginseng Radix Rubra, $C$. officinalis Sieb. et Zucc., L. chinense Mill, D. tenuipes, and C. longa Linn, showed significant improvements in EF and intercourse satisfaction, as measured by the IEF in patients with ED. Further studies on a larger number of patients in the long term should be conducted to confirm the hormonal and hemodynamic changes and the related symptom improvement induced by KBMSI-2.

\section{CONFLICTS OF INTEREST}

The authors have nothing to disclose.

\section{REFERENCES}

1. Lue TF. Erectile dysfunction. N Engl J Med 2000;342:1802-13.

2. Carvalheira AA, Pereira NM, Maroco J, Forjaz V. Dropout in the treatment of erectile dysfunction with PDE5: a study on predictors and a qualitative analysis of reasons for discontinuation. J Sex Med 2012;9:2361-9.

3. Salonia A, Gallina A, Zanni G, Briganti A, Dehò $F$, Saccà A, et al. Acceptance of and discontinuation rate from erectile dysfunction oral treatment in patients following bilateral nervesparing radical prostatectomy. Eur Urol 2008;53:564-70.

4. Ljunggren C, Hedelin H, Salomonsson K, Ströberg P. Giving patients with erectile dysfunction the opportunity to try all three available phosphodiesterase type 5 inhibitors contributes to better long-term treatment compliance. J Sex Med 2008; 5:469-75.

5. Raina R, Lakin MM, Agarwal A, Sharma R, Goyal KK, Montague $\mathrm{DK}$, et al. Long-term effect of sildenafil citrate on erectile dysfunction after radical prostatectomy: 3-year follow-up. Urology 2003;62:110-5.

6. Leungwattanakij S, Flynn V Jr, Hellstrom WJ. Intracavernosal injection and intraurethral therapy for erectile dysfunction. Urol Clin North Am 2001;28:343-54

7. Sundaram CP, Thomas W, Pryor LE, Sidi AA, Billups K, Pryor JL. Long-term follow-up of patients receiving injection therapy for erectile dysfunction. Urology 1997;49:932-5.

8. Vardi Y, Sprecher E, Gruenwald I. Logistic regression and survival analysis of 450 impotent patients treated with injection therapy: long-term dropout parameters. J Urol 2000;163:46770.

9. Fode M, Ohl DA, Ralph D, Sønksen J. Penile rehabilitation after radical prostatectomy: what the evidence really says. BJU Int 2013;112:998-1008.

10. Montorsi F, Brock G, Stolzenburg JU, Mulhall J, Moncada I, Patel HR, et al. Effects of tadalafil treatment on erectile function recovery following bilateral nerve-sparing radical prostatectomy: a randomised placebo-controlled study (REACTT). Eur Urol 2014;65:587-96.

11. Park CS, Ryu SD, Hwang SY. Elevation of intracavernous pressure and NO-cGMP activity by a new herbal formula in penile tissues of aged and diabetic rats. J Ethnopharmacol 2004;94:8592.

12. Yoon BI, Hong C, Lee JH, Kim SJ, Kim HS, Ha US, et al. The effects of new herbal formula (KBMSI-2) on penile erection and expression of nitric oxide synthase isoforms in streptozotocin-induced diabetic rat model. Chin J Integr Med 2013 Nov 16 [Epub]. https://doi.org/10.1007/s11655-013-1546-z.

13. Rosen RC, Cappelleri JC, Gendrano N 3rd. The International Index of Erectile Function (IIEF): a state-of-the-science review. Int J Impot Res 2002;14:226-44.

14. Morley JE, Charlton E, Patrick P, Kaiser FE, Cadeau P, McCready D, et al. Validation of a screening questionnaire for androgen deficiency in aging males. Metabolism 2000;49:123942.

15. Daig I, Heinemann LA, Kim S, Leungwattanakij S, Badia X, Myon E, et al. The Aging Males' Symptoms (AMS) scale: review of its methodological characteristics. Health Qual Life Outcomes 2003;1:77.

16. Kam SC, Choi SM, Jeh SU, Lee SH, Hwa JS, Jung KH, et al. Efficacy and safety of a herbal formula that mainly consists of cornus officinalis for erectile dysfunction: a double-blind, 
placebo-controlled study. Korean J Urol 2007;48:741-7.

17. Chauhan NS, Sharma V, Dixit VK, Thakur M. A review on plants used for improvement of sexual performance and virility. Biomed Res Int 2014;2014:868062.

18. Xiong G, Li B, Wang K, Li H. Chinese herb formulae for treatment of erectile dysfunction: a systematic review of randomised controlled clinical trials. Andrologia 2014;46:201-23.

19. Choi HK, Seong DH, Rha KH. Clinical efficacy of Korean red ginseng for erectile dysfunction. Int J Impot Res 1995;7:181-6.

20. Hong B, Ji YH, Hong JH, Nam KY, Ahn TY. A double-blind crossover study evaluating the efficacy of Korean red ginseng in patients with erectile dysfunction: a preliminary report. J Urol 2002;168:2070-3.

21. de Andrade E, de Mesquita AA, Claro Jde A, de Andrade PM, Ortiz V, Paranhos M, et al. Study of the efficacy of Korean red ginseng in the treatment of erectile dysfunction. Asian J Androl 2007;9:241-4.

22. Choi YD, Xin ZC, Choi HK. Effect of Korean red ginseng on the rabbit corpus cavernosal smooth muscle. Int J Impot Res 1998;10:37-43.

23. McKay D. Nutrients and botanicals for erectile dysfunction: examining the evidence. Altern Med Rev 2004;9:4-16.

24. Kim HJ, Woo DS, Lee G, Kim JJ. The relaxation effects of ginseng saponin in rabbit corporal smooth muscle: is it a nitric oxide donor? Br J Urol 1998;82:744-8.

25. Abdel Aziz MT, El Asmer MF, Rezq A, Kumosani TA, Mostafa S, Mostafa T, et al. Novel water-soluble curcumin derivative mediating erectile signaling. Sex Med 2010;7:2714-22.

26. Abdel Aziz MT, Motawi T, Rezq A, Mostafa T, Fouad HH, Ahmed $\mathrm{HH}$, et al. Effects of a water-soluble curcumin protein conjugate vs. pure curcumin in a diabetic model of erectile dysfunction. J Sex Med 2012;9:1815-33.

27. Abdel Aziz MT, Rezq AM, Atta HM, Fouad H, Zaahkouk AM, Ahmed HH, et al. Molecular signalling of a novel curcumin derivative versus Tadalafil in erectile dysfunction. Andrologia 2015;47:616-25.

28. Zaahkouk AM, Abdel Aziz MT, Rezq AM, Atta HM, Fouad $\mathrm{HH}$, Ahmed $\mathrm{HH}$, et al. Efficacy of a novel water-soluble curcumin derivative versus sildenafil citrate in mediating erectile function. Int J Impot Res 2015;27:9-15.

29. Chen MF, Tsai JT, Chen LJ, Wu TP, Yang JJ, Yin LT, et al. Antihypertensive action of allantoin in animals. Biomed Res Int 2014;2014:690135.

30. Liu K, Xu H, Lv G, Liu B, Lee MK, Lu C, et al. Loganin attenuates diabetic nephropathy in C57BL/6J mice with diabetes induced by streptozotocin and fed with diets containing high level of advanced glycation end products. Life Sci 2015;123:7885. 